\title{
Irregular Distributed Space-Time Code Design for Near-Capacity Cooperative Communications
}

\author{
Lingkun Kong, Soon Xin Ng, Robert G. Maunder, and Lajos Hanzo \\ School of ECS, University of Southampton, SO17 1BJ, United Kingdom. \\ Tel: +44-23-8059 3125, Fax: +44-23-8059 4508 \\ Email: \{lk06r,sxn,rm,lh\}@ecs.soton.ac.uk, http://www-mobile.ecs.soton.ac.uk
}

\begin{abstract}
This paper presents an Irregular Distributed Hybrid Concatenated Space-Time (Ir-DHC-ST) coding scheme designed for nearcapacity cooperative communications. A serial concatenated scheme comprising an IRregular Convolutional Code (IRCC), a recursive UnityRate Code (URC) and a Space-Time Block Code (STBC) was designed for the sake of approaching the corresponding source-to-relay link capacity, where the IRCC was optimized with the aid of EXtrinsic Information Transfer (EXIT) charts which was used at the source node. At the relay node, another IRCC is concatenated serially with an identical STBC. The relay's IRCC is re-optimized based on EXIT chart analysis for the sake of approaching the relay network's capacity, before transmitting the relayed information. We will demonstrate that the topology of the IrDHC-ST system coincides with that of a Distributed Turbo Code (DTC). At the destination node, a novel three-stage iterative decoding scheme is constructed in order to achieve decoding convergence to an infinitesimally low Bit Error Ratio (BER). Finally, it is shown that our joint sourceand-relay mode design based on EXIT chart analysis is capable of nearcapacity cooperative communications.
\end{abstract}

\section{INTRODUCTION}

In the past few years, cooperative communication schemes [1][3] have been intensively studied, which combine the benefits of distributed Multiple-Input Multiple-Output (MIMO) systems with relay technologies. In a relay network, where the nodes (users) are equipped with either single or multiple antennas, cooperative communications allow the nodes (users) to assist each other in forwarding (relay) all messages to the destination, rather than transmitting only their own messages. Since the MIMO transmitter's elements in such a network are distributed, the network forms a "distributed MIMO" system. For the sake of improving the diversity gain of relay-aided networks, numerous cooperative protocols [1]-[4] have been proposed. In most cooperative scenarios, predominantly the Decode-and-Forward (DF) and Amplify-and-Forward (AF) protocols are used. However, a strong channel code is required for mitigating the potential error propagation in the DF scheme or to avoid the noise-enhancement in the AF scheme.

Inspired by the classic turbo codes used in non-cooperative communication scenarios, Distributed Turbo Codes (DTC) [5] have been proposed for "distributed MIMO" systems, which benefit from a turbo processing gain. However, DTCs suffer from having an imperfect communication link between the source and relay nodes. Hence a three-component Distributed Turbo Trellis Coded Modulation (DTTCM) scheme has been proposed in [6], which takes into consideration the realistic condition of having an imperfect source-torelay communication link. The DTTCM scheme [6] designed using EXtrinsic Information Transfer (EXIT) chart analysis [7], [8] is capable of minimizing the decoding error probability at the relay node and performs close to its idealized counterpart that assumes perfect decoding at the relay node.

Against this background, our objective here is to design a cooperative scheme, which is capable of performing close to the corresponding Discrete-input Continuous-output Memoryless Channel (DCMC) [9], [10] capacity of the cooperative network, rather than that of non-cooperative communication scenarios [11], [12]. For the sake of approaching the DCMC capacity of the cooperative network, a

The financial support of the China-UK Scholarship Council, as well as that of the EPSRC UK, the EU under the auspices of the Optimix project is gratefully acknowledged. new Irregular Distributed Hybrid Concatenated Space-Time (Ir-DHCST) coding scheme is proposed, where two IRregular Convolutional Codes (IRCC) [13], [14] having different IRCC weighting coefficients are employed for near-capacity cooperative communications at the source and relay nodes, respectively.

The rest of this paper is organised as follows. The system model is described in Section II. Section III specifies the encoding and decoding processes of the novel Ir-DHC-ST coding scheme. The network's DCMC capacity computation and the EXIT chart aided joint source-and-relay mode design are detailed in Section IV, while our simulation results and discussions are provided in Section V. Finally, we conclude in Section VI.

\section{SYSTEM MODEL}

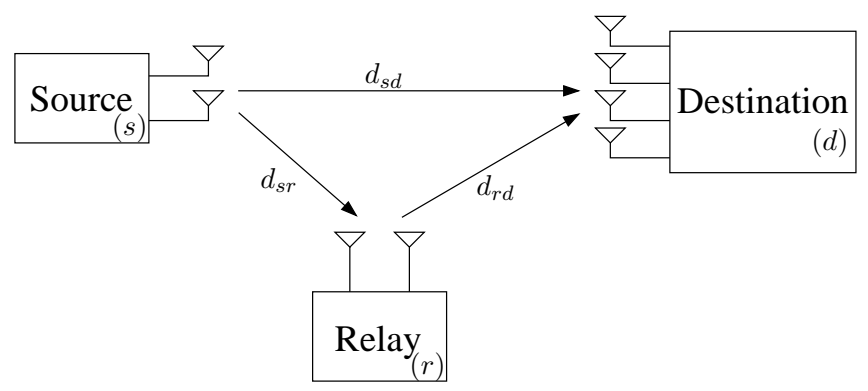

Fig. 1. Schematic of a single relay aided multi-antenna system, where $d_{a b}$ is the geographical distance between node $a$ and node $b$.

We consider a two-hop relay-aided network, which has a single source node having $N_{s}=2$ antennas, a single relay node equipped with $N_{r}=2$ antennas and a destination node having $N_{d}=4$ antennas, where all nodes obey the half-duplex constraint, i.e. a node cannot transmit and receive simultaneously. The schematic of the proposed system is shown in Fig. 1, which typically entails two phases. In Phase I, the source node $(s)$ broadcasts the information both to the relay node $(r)$ and to the destination node $(d)$. The relay node $(r)$ processes the received information and forwards it to the destination node $(d)$ in Phase II, while the source node $(s)$ remains silent. The destination performs decoding based on the messages it received in both phases. As in [15], we model the communication links in Fig. 1 as being subjected to both long-term free-space path loss as well as to short-term uncorrelated Rayleigh fading.

Let $d_{a b}$ denote the geometrical distance between nodes $a$ and $b$. The path loss between these nodes can be modeled by [15]:

$$
P_{a b}=\frac{K}{d_{a b}^{\alpha}},
$$

where $K$ is a constant that depends on the environment and $\alpha$ is the path-loss exponent. For a free space scenario, we have $\alpha=2$. The relationship between the energy $E_{s r}$ received at the relay node and that of the destination node $E_{s d}$ can be expressed as:

$$
E_{s r}=\frac{P_{s r}}{P_{s d}} E_{s d}=G_{s r} E_{s d}
$$

where $G_{s r}$ is the power-gain (or geometrical-gain) [15] experienced by the source-to-relay link with respect to the source-to-destination 
link as a benefit of the commensurately reduced distance and path loss, which can be expressed as:

$$
G_{s r}=\left(\frac{d_{s d}}{d_{s r}}\right)^{2} .
$$

Similarly, the power-gain at the relay-to-destination link with respect to the source-to-destination link can be formulated as:

$$
G_{r d}=\left(\frac{d_{s d}}{d_{r d}}\right)^{2}
$$

Naturally, the power-gain at the source-to-destination link with respect to itself is unity, i.e. $G_{s d}=1$. Therefore, according to the geometry of Fig. 1, the power-gains $G_{s r}$ and $G_{r d}$ have to satisfy:

$$
G_{s r}+G_{r d}+2 \sqrt{G_{s r} G_{r d}} \geq G_{s r} G_{r d}
$$

We assume that the relay node is closer to the source node than to the destination node, while both the source and relay are far away from the destination, namely we have $G_{s r}>G_{r d}$. In this scenario, the relay benefits from a higher received signal power than the destination. This assumption facilitates the employment of nearperfect DF relaying (similar arguments can be found in [16], [17]), otherwise other relaying modes might be better choices (e.g. Amplifyand-Forward (AF) and Compress-and-Forward (CF)). Hence, the vector hosting the received signal at the relay node during Phase $I$, which consists of $L_{S}$ number of symbol periods, can be formulated as:

$$
\mathbf{y}_{r}=\sqrt{G_{s r}} \mathbf{H}_{r} \mathbf{c}_{s}+\mathbf{n}_{r},
$$

where $\mathbf{y}_{r}=\left[y_{r, 1}, \ldots, y_{r, N_{r}}\right]^{T}$ is the $N_{r}$-element vector of the received signals at the relay node, $\mathbf{H}_{r}$ is the $\left(N_{r} \times N_{S}\right)$-element channel matrix, the entries of which are independent and identically complex Gaussian distributed with a zero mean and a variance of 0.5 per dimension. Furthermore, $\mathbf{c}_{s}=\left[c_{s, 1}, \ldots, c_{s, N_{s}}\right]^{T}$ is an $N_{s}$-element vector of the signals transmitted from the source node and $\mathbf{n}_{r}=\left[n_{r, 1}, \ldots, n_{r, N_{r}}\right]^{T}$ is an $N_{r}$-element noise vector. Each element of $\mathbf{n}_{r}$ is an Additive White Gaussian Noise (AWGN) process having a zero mean and a variance of $N_{0} / 2$ per dimension. By contrast, the signal vector received at the destination node during Phase $I$ can be expressed as:

$$
\mathbf{y}_{d}^{I}=\sqrt{G_{s d}} \mathbf{H}_{d}^{I} \mathbf{c}_{s}+\mathbf{n}_{d},
$$

and the signal vector received at the destination node during Phase $I I$, when $L_{r}$ number of symbols are transmitted from the relay node, is formulated as:

$$
\mathbf{y}_{d}^{I I}=\sqrt{G_{r d}} \mathbf{H}_{d}^{I I} \mathbf{c}_{r}+\mathbf{n}_{d}
$$

where $\mathbf{y}_{d}^{I}=\left[y_{d, 1}^{I}, \ldots, y_{d, N_{d}}^{I}\right]^{T}$ and $\mathbf{y}_{d}^{I I}=\left[y_{d, 1}^{I I}, \ldots, y_{d, N_{d}}^{I I}\right]^{T}$ are both $N_{d^{-}}$ element vectors of the signals received during Phase $I$ and Phase $I I$ at the destination node, $\mathbf{H}_{d}^{I} \in \mathbb{C}^{N_{d} \times N_{s}}$ and $\mathbf{H}_{d}^{I I} \in \mathbb{C}^{N_{d} \times N_{r}}$ are the corresponding channel matrices, the entries of which are independent and identically complex Gaussian distributed with a zero mean and a variance of 0.5 per dimension. Finally, $\mathbf{c}_{r}=\left[c_{r, 1}, \ldots, c_{r, N_{r}}\right]^{T}$ is an $N_{r}$-element vector of the signals transmitted from the relay node and $\mathbf{n}_{d}=\left[n_{d, 1}, \ldots, n_{d, N_{d}}\right]^{T}$ is an $N_{d}$-element AWGN vector with each element having a zero mean and a variance of $N_{0} / 2$ per dimension.

\section{IRREgular Distributed Hybrid CONCATENATED SPACE-TIME CODES}

\section{A. Ir-DHC-ST Encoder}

At the source node of the proposed two-hop relay-aided network, we use Alamouti's STBC [18], which is amalgamated with a recursive Unity-Rate Code (URC) ${ }^{1}$ as the inner code, while an IRCC [13], [14] with an average code rate of $R_{S}$ is employed as the outer code for the

${ }^{1}$ URCs were proposed by Divsalar et al. [19] for the sake of extending the overall system's impulse response to an infinite duration, which efficiently spreads the extrinsic information, hence improves the achievable iterative detection gain.

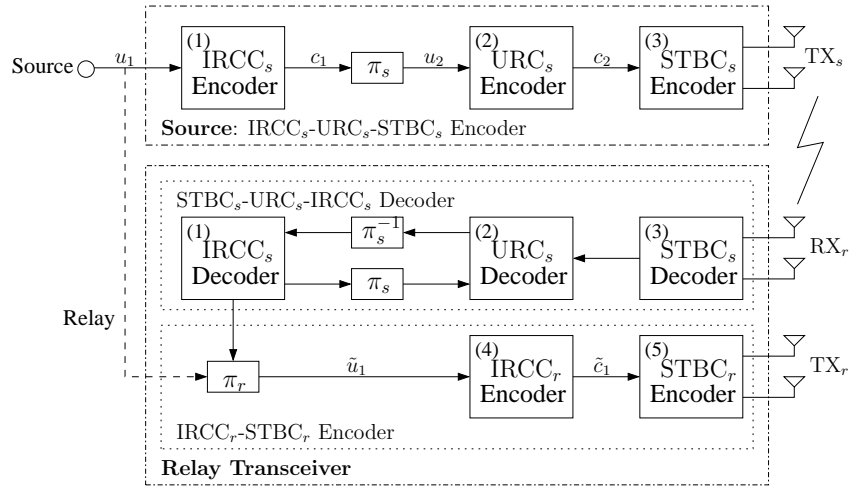

Fig. 2. Schematic of the Irregular Distributed Hybrid Concatenated SpaceTime (Ir-DHC-ST) encoder where the dashed line indicates the error-free decoding at the relay node.

sake of achieving a performance that is close to the capacity of the source-to-relay link. On the other hand, at the relay node, another IRCC having a code rate of $R_{r}$ is amalgamated with Alamouti's STBC scheme for the sake of achieving a performance that is close to the relay network's capacity. The notations $\pi_{s}$ and $\pi_{r}$ in Fig. 2 represent the bit-wise random interleaver used at the source node and the relay node, respectively. If the relay is capable of decoding the signals received from the source node without errors in Phase $I$, the relay-aided scheme may be referred to as an Irregular Hybrid Concatenated Space-Time (Ir-HC-ST) coding arrangement. Hence, we could view this relay-aided system as an Irregular Distributed Hybrid Concatenated Space-Time (Ir-DHC-ST) coding scheme, as depicted in Fig. 2, where the dashed line indicates the error-free decoding at the relay node.

\section{B. Three-Stage Iterative Decoding at the Destination}

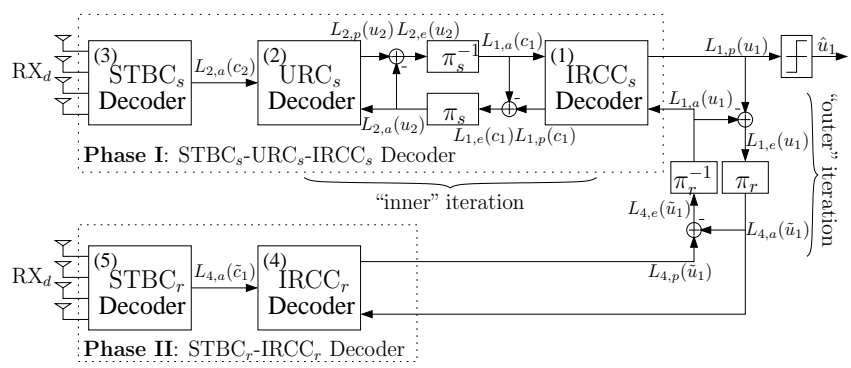

Fig. 3. The three-stage iterative decoder of the Irregular Distributed Hybrid Concatenated Space-Time (Ir-DHC-ST) code.

The novel three-stage iterative decoder structure of the Ir-DHCST scheme is illustrated in Fig. 3. At the destination node, the signals received during Phase $I$ are first decoded by the amalgamated ${ }^{2}$ " $\mathrm{STBC}_{s}-\mathrm{URC}_{s}$ " decoder in order to produce the a priori LogLikelihood Ratio (LLR) values $L_{1, a}\left(c_{1}\right)$ of the coded bits $c_{1}$ by the Maximum Aposteriori Probability (MAP) algorithm [20]. The $\mathrm{IRCC}_{s}$ decoder seen at the top right of Fig. 3 processes the information forwarded by the " $\mathrm{STBC}_{s}-\mathrm{URC}_{s}$ " decoder in conjunction with the $a$ priori LLR values $L_{1, a}\left(u_{1}\right)$ of the information bits $u_{1}$ gleaned by the "STBC $r$-IRCC $r$ " relay-decoder in order to generate the a posteriori LLR values $L_{1, p}\left(u_{1}\right)$ and $L_{1, p}\left(c_{1}\right)$ of the information bits $u_{1}$ and of the coded bits $c_{1}$, respectively. As seen in Fig. 3, the a priori LLRs $L_{1, a}\left(c_{1}\right)$ are subtracted from the a posteriori LLR values $L_{1, p}\left(c_{1}\right)$ and then they are fed back to the "STBC ${ }_{s}-\mathrm{URC}_{s}$ " decoder as the a priori information $L_{2, a}\left(u_{2}\right)$ through the interleaver $\pi_{s}$. We term this information-exchange process seen in the top trace of Fig. 3

${ }^{2}$ The terminology "amalgamated" is used here to indicate that although it might have some benefits to exchange extrinsic information between these two blocks, no iterations were used between them. 
as the "inner" iteration ${ }^{3}$. Similarly, during the "outer" iterations, the a priori LLR values $L_{1, a}\left(u_{1}\right)$ fed into the $\operatorname{IRCC}_{s}$ decoder are also subtracted from the a posteriori LLR values $L_{1, p}\left(u_{1}\right)$ for the sake of generating the extrinsic LLR values $L_{1, e}\left(u_{1}\right)$ as seen at the top right corner of Fig. 3. Then $L_{1, e}\left(u_{1}\right)$ is passed to the amalgamated "STBC $r^{-}$ IRCC $_{r}$ " relay-decoder as the a priori information $L_{4, a}\left(\tilde{u}_{1}\right)$ through the interleaver $\pi_{r}$ in conjunction with the signals received during Phase II in order to compute the a posteriori LLR values $L_{4, p}\left(\tilde{u}_{1}\right)$ of the permuted information bits $\tilde{u}_{1}$ from the relay node. As seen in Fig. 3 , the extrinsic information $L_{4, e}\left(\tilde{u}_{1}\right)$ is generated by subtracting the a priori information $L_{4, a}\left(\tilde{u}_{1}\right)$ from the a posteriori information $L_{4, p}\left(\tilde{u}_{1}\right)$, before $L_{4, e}\left(\tilde{u}_{1}\right)$ is fed back to the $\operatorname{IRCC}_{s}$ decoder as the a priori information $L_{1, a}\left(u_{1}\right)$ through the de-interleaver $\pi_{r}^{-1}$. During the last "outer" iteration, only the LLR values $L_{1, p}\left(u_{1}\right)$ of the original information bits $u_{1}$ are required, which are passed to the hard-decision block of Fig. 3 in order to estimate the source bits.

\section{NEAR-CAPACITY SYSTEM DESIGN AND ANALYSIS}

In order to design a near-capacity coding system for the proposed two-hop relay-aided network, we first derive the network's DCMC [9], [10] capacity formula for Alamouti's STBC scheme in Section IV-A. Then, the EXIT chart based joint source-and-relay mode design will be carried out in Section IV-B.

\section{A. Network Capacity}

We approximate the two-hop relay-aided network capacity as the maximum achievable rate attained during the transmission of the source node in Phase $I$ and an independent transmission by the relay node in Phase II. In this contribution, we employ Alamouti's G2 scheme [18] at both the source and relay nodes, whose codeword matrix is given by:

$$
\mathbf{C}_{G 2}=\left(\begin{array}{cc}
c_{1} & c_{2} \\
-\bar{c}_{2} & \bar{c}_{1}
\end{array}\right)^{T}
$$

Based on Eqs. (7) and (8), the signal received at the destination node during $V=2$ consecutive symbol periods in Phase I and Phase II can be written as:

$$
\mathbf{Y}_{d}^{I}=\sqrt{G_{s d}} \mathbf{H}_{d}^{I} \mathbf{C}_{s}+\mathbf{N}_{d}
$$

and

$$
\mathbf{Y}_{d}^{I I}=\sqrt{G_{r d}} \mathbf{H}_{d}^{I I} \mathbf{C}_{r}+\mathbf{N}_{d}
$$

respectively, where $\mathbf{Y}_{d}^{I}=\left[\mathbf{y}_{d, 1}^{I}, \ldots, \mathbf{y}_{d, V}^{I}\right] \in \mathbb{C}^{N_{d} \times V}$ and $\mathbf{Y}_{d}^{I I}=$ $\left[\mathbf{y}_{d, 1}^{I I}, \ldots, \mathbf{y}_{d, V}^{I I}\right] \in \mathbb{C}^{N_{d} \times V}$ are the matrices hosting the sampled signal received during Phase $I$ and Phase II at the destination node, while $\mathbf{H}_{d}^{I} \in \mathbb{C}^{N_{d} \times N_{s}}$ and $\mathbf{H}_{d}^{I I} \in \mathbb{C}^{N_{d} \times N_{r}}$ are the corresponding quasi-static channel matrices, which are constant over $V=2$ consecutive symbol periods in Phase $I$ and Phase $I I$, respectively. Furthermore, $\mathbf{C}_{s}=$ $\left[\mathbf{c}_{s, 1}, \ldots, \mathbf{c}_{s, V}\right] \in \mathbb{C}^{N_{s} \times V}$ and $\mathbf{C}_{r}=\left[\mathbf{c}_{r, 1}, \ldots, \mathbf{c}_{r, V}\right] \in \mathbb{C}^{N_{r} \times V}$ represent Alamouti's $\mathrm{G} 2$ matrices characterizing the transmissions of the source and relay nodes, while $\mathbf{N}_{d}=\left[\mathbf{n}_{d, 1}, \ldots, \mathbf{n}_{d, V}\right] \in \mathbb{C}^{N_{d} \times V}$ represents the AWGN matrix incurred during PhaseI and PhaselI.

When complex-valued $\mathcal{M}$-ary PSK/QAM signalling is employed, we have a total of $M=\mathcal{M}^{2}$ possible G2 codeword matrix combinations for $V=2$ consecutive symbol periods. Based on Eqs. (10) and (11), the conditional probability of receiving a signal hosted by the matrix $\mathbf{Y}$, given that an $M$-ary $\mathrm{G} 2$ codeword matrix $\mathbf{C}^{m}$, $m \in\{1, \ldots, M\}$, was transmitted over the channel subjected to path

\footnotetext{
${ }^{3}$ Explicitly, at the destination node, the extrinsic information exchange between the amalgamated " $\mathrm{STBC}_{s}-\mathrm{URC}_{s}$ " decoder and the $\mathrm{IRCC}_{s}$ decoder is referred to as the "inner" iteration, while that between the parallel amalgamated " $\mathrm{STBC}_{s}-\mathrm{URC}_{s}-\mathrm{IRCC}_{s}$ " decoder and the amalgamated "STBC ${ }_{r}$ $\mathrm{IRCC}_{r}$ " relay-decoder is referred to as the "outer" iteration.
}

loss and short-term uncorrelated flat Rayleigh fading is determined by the Probability Density Function (PDF) of the noise, yielding:

$$
\begin{array}{r}
p^{I}\left(\mathbf{Y}_{d}^{I} \mid \mathbf{C}_{s}^{m}\right)=\frac{1}{\left(\pi N_{0}\right)^{V N_{d}}} \exp \left(\frac{-\left\|\mathbf{Y}_{d}^{I}-\sqrt{G_{s d}} \mathbf{H}_{d}^{I} \mathbf{C}_{s}^{m}\right\|^{2}}{N_{0}}\right), \\
=\prod_{k=1}^{V} \prod_{n=1}^{N_{d}} \frac{1}{\pi N_{0}} \exp \left(\frac{-\left|y_{n, k}^{I}-\sum_{t=1}^{N_{s}} \sqrt{G_{s d}} h_{n, t}^{I} c_{s, t, k}^{m}\right|^{2}}{N_{0}}\right),
\end{array}
$$

and

$$
\begin{array}{r}
p^{I I}\left(\mathbf{Y}_{d}^{I I} \mid \mathbf{C}_{r}^{m}\right)=\frac{1}{\left(\pi N_{0}\right)^{V N_{d}}} \exp \left(\frac{-\left\|\mathbf{Y}_{d}^{I I}-\sqrt{G_{r d}} \mathbf{H}_{d}^{I I} \mathbf{C}_{r}^{m}\right\|^{2}}{N_{0}}\right), \\
=\prod_{k=1}^{V} \prod_{n=1}^{N_{d}} \frac{1}{\pi N_{0}} \exp \left(\frac{-\left|y_{n, k}^{I I}-\sum_{t=1}^{N_{r}} \sqrt{G_{r d}} h_{n, t}^{I I} c_{r, t, k}^{m}\right|^{2}}{N_{0}}\right),
\end{array}
$$

during Phase I and Phase II, respectively. The two-hop relayaided network's capacity per symbol period evaluated for Alamouti's G2 scheme using complex-valued $\mathscr{M}$-ary PSK/QAM signalling for transmission over the DCMC can be shown to be:

$$
\begin{aligned}
& C_{\mathrm{DCMC}}^{\text {Coop-G2 }}= \frac{1}{V} \cdot \frac{L_{s}}{L_{s}+L_{r}} \cdot \max _{p\left(\mathbf{C}_{s}^{1}\right) \ldots p\left(\mathbf{C}_{s}^{M}\right)} \sum_{m=1}^{M} \int_{\mathbf{Y}_{d}^{I}} p^{I}\left(\mathbf{Y}_{d}^{I} \mid \mathbf{C}_{s}^{m}\right) p\left(\mathbf{C}_{s}^{m}\right) \\
& \cdot \log _{2}\left(\frac{p^{I}\left(\mathbf{Y}_{d}^{I} \mid \mathbf{C}_{s}^{m}\right)}{\sum_{n=1}^{M} p^{I}\left(\mathbf{Y}_{d}^{I} \mid \mathbf{C}_{s}^{n}\right) p\left(\mathbf{C}_{s}^{n}\right)}\right) d \mathbf{Y}_{d}^{I} \\
&+\frac{1}{V} \cdot \frac{L_{r}}{L_{s}+L_{r}} \cdot \max _{p\left(\mathbf{C}_{r}^{1}\right) \ldots p\left(\mathbf{C}_{r}^{M}\right)} \sum_{m=1}^{M} \int_{\mathbf{Y}_{d}^{I I}} p^{I I}\left(\mathbf{Y}_{d}^{I I} \mid \mathbf{C}_{r}^{m}\right) p\left(\mathbf{C}_{r}^{m}\right) \\
& \cdot \log _{2}\left(\frac{p^{I I}\left(\mathbf{Y}_{d}^{I I} \mid \mathbf{C}_{r}^{m}\right)}{\sum_{n=1}^{M} p^{I I}\left(\mathbf{Y}_{d}^{I I} \mid \mathbf{C}_{r}^{n}\right) p\left(\mathbf{C}_{r}^{n}\right)}\right) d \mathbf{Y}_{d}^{I I},
\end{aligned}
$$

where the right hand side of Eq. (14) is maximized, when we have $p\left(\mathbf{C}_{s}^{m}\right)=p\left(\mathbf{C}_{r}^{m}\right)=1 / M$ for $m \in\{1, \ldots, M\}$. Hence, Eq. (14) can be simplified to:

$$
\begin{aligned}
C_{\text {DCMC }}^{\text {Coop-G2 }}= & \frac{\log _{2}(M)}{V}- \\
& \frac{L_{s}}{M V\left(L_{s}+L_{r}\right)} \sum_{m=1}^{M} E\left[\log _{2} \sum_{n=1}^{M} \exp \left(\Psi_{m, n}^{I}\right) \mid \mathbf{C}_{s}^{m}\right]- \\
& \frac{L_{r}}{M V\left(L_{s}+L_{r}\right)} \sum_{m=1}^{M} E\left[\log _{2} \sum_{n=1}^{M} \exp \left(\Psi_{m, n}^{I I}\right) \mid \mathbf{C}_{r}^{m}\right],(15)
\end{aligned}
$$

where $E\left[A \mid \mathbf{C}^{m}\right]$ is the expectation of $A$ conditioned on $\mathbf{C}^{m}$ and the expectations in Eq. (15) are taken over $\mathbf{H}_{d}^{I}, \mathbf{H}_{d}^{I I}$ and $\mathbf{N}_{d}$, while $\Psi_{m, n}^{I}$ and $\Psi_{m, n}^{I I}$ are given by:

$$
\Psi_{m, n}^{I}=\frac{-\left\|\sqrt{G_{s d}} \mathbf{H}_{d}^{I}\left(\mathbf{C}_{s}^{m}-\mathbf{C}_{s}^{n}\right)+\mathbf{N}_{d}\right\|^{2}+\left\|\mathbf{N}_{d}\right\|^{2}}{N_{0}}
$$

and

$$
\Psi_{m, n}^{I I}=\frac{-\left\|\sqrt{G_{r d}} \mathbf{H}_{d}^{I I}\left(\mathbf{C}_{r}^{m}-\mathbf{C}_{r}^{n}\right)+\mathbf{N}_{d}\right\|^{2}+\left\|\mathbf{N}_{d}\right\|^{2}}{N_{0}},
$$

respectively. Based on the relay-aided network's DCMC capacity formula of Eq. (15) we can evaluate the capacity by substituting the corresponding G2 codeword matrices $\mathbf{C}_{s} \in \mathbb{C}^{N_{s} \times V}$ and $\mathbf{C}_{r} \in \mathbb{C}^{N_{r} \times V}$ into Eqs. (16) and (17), respectively.

The resultant bandwidth efficiency is determined by normalising the relay-aided network's capacity given by Eq. (15), with respect to the product of the bandwidth $W$ and the signalling period $T$ :

$$
\eta=\frac{C}{W T}[\mathrm{bit} / \mathrm{s} / \mathrm{Hz}]
$$

where $W T=1$ for PSK/QAM schemes, when assuming zero Nyquist excess bandwidth. The bandwidth efficiency, $\eta$, is typically plotted against the Signal-to-Noise Ratio (SNR) per bit given by $E_{b} / N_{0}=$ $\mathrm{SNR} / \eta$. For simplicity, we will refer to $\eta$ as the network's capacity. 


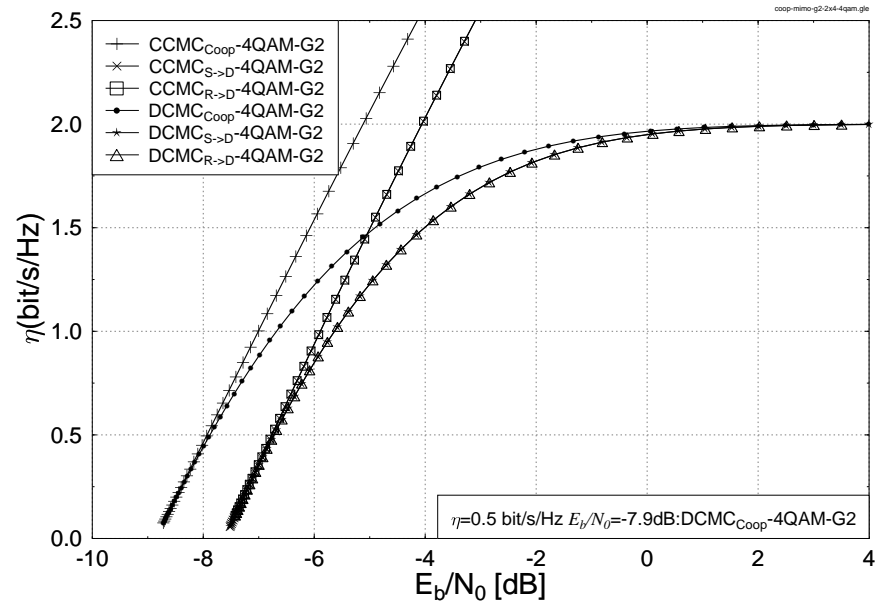

Fig. 4. The network capacity of the two-hop relay-aided system employing Alamouti's space-time block code when the network is configured with $N_{s}=2$, $N_{r}=2$ and $N_{d}=4$ as well as $L_{s}=L_{r}$.

\section{B. Joint Code Design for the Source-and-Relay Nodes Based on EXIT Charts}

For the sake of analysing the convergence behaviour of the three-stage iterative decoder of the two-hop relay-aided system, we use two EXIT charts [7], [8] to examine the evolution of the input/output mutual information exchanges between the inner amalgamated "STBC $\mathrm{STRC}_{s}$ " decoder and the outer $\mathrm{IRCC}_{s}$ decoder at the relay node, as well as between the parallel amalgamated " $\mathrm{STBC}_{S^{-}}$ $\mathrm{URC}_{s}-\mathrm{IRCC}_{s}$ " decoder and the amalgamated "STBC $r$-IRCC $r$ " decoder at the destination node during the consecutive iterations. As has been investigated in [11], [12], a narrow but marginally open EXIT-tunnel between the EXIT curves of the inner amalgamated " $\mathrm{STBC}_{s}-\mathrm{URC}_{s}$ " decoder and the outer $\mathrm{IRCC}_{s}$ decoder at the relay node indicates that a performance close to the capacity of the source-to-relay link could be achieved. Similarly, at the destination node, a narrow-but-open EXIT-tunnel between the EXIT curves of the parallel amalgamated " $\mathrm{STBC}_{s}-\mathrm{URC}_{s}-\mathrm{IRCC}_{s}$ " decoder and the amalgamated "STBC -IRCC $_{r}$ " decoder indicates the possibility of achieving decoding convergence to an infinitesimally low Bit Error Ratio (BER) at SNRs close to the relay-aided network's capacity. Therefore, the joint source-and-relay mode design procedure can be simplified to two EXIT curve matching problems, which is summarised as follows:

Step 1: Choose a specific average code rate $R_{S}$ for the $\mathrm{IRCC}_{S}$ at the source node and employ the EXIT curve matching algorithm of [13] to obtain the optimized weighting coefficients $\alpha_{i}, i=1, \ldots, 17$ of $\mathrm{IRCC}_{s}$, where a narrow but marginally open EXIT-tunnel between the EXIT curves of the inner amalgamated " $\mathrm{STBC}_{s}-\mathrm{URC}_{s}$ " decoder and the outer $\mathrm{IRCC}_{s}$ decoder emerges at the relay node. This implies that a near-capacity performance may be achieved for the source-torelay communication link.

Step 2: Fix the optimized weighting coefficients $\alpha_{i}, i=1, \ldots, 17$ of the $\mathrm{IRCC}_{s}$ obtained in Step $\mathbf{1}$ at the source node, perform iterative decoding by exchanging extrinsic information between the amalgamated "STBC ${ }_{s}-\mathrm{URC}_{s}$ " decoder and the $\mathrm{IRCC}_{s}$ decoder during Phase $I$ at the destination node, until the further increase of the area $A_{E}$ under the EXIT curve of the amalgamated " $\mathrm{STBC}_{s}-\mathrm{URC}_{s}-\mathrm{IRCC}_{s}$ " decoder becomes marginal, then stop this "inner" iterative decoding process.

Step 3: Assume perfectly error-free DF relaying at the relay node in the second EXIT chart analysis used for designing the Ir-DHCST coding system. Use the same EXIT curve matching algorithm of [13] to match the SNR-dependent EXIT curve of the amalgamated "STBC ${ }_{r}$-IRCC ${ }_{r}$ " decoder to the target EXIT curve of the amalgamated " $\mathrm{STBC}_{s}-\mathrm{URC}_{s}-\mathrm{IRCC}_{s}$ " decoder observed in Step 2. Obtain the maximized average code rate $R_{r}$ and the optimized weighting coefficients $\beta_{j}, j=1, \ldots, 17$ of IRCC $_{r}$ when a narrow-but-open EXITtunnel emerges, which indicates the possibility of achieving decoding convergence to an infinitesimally low BER at an SNR near the relayaided network's capacity.

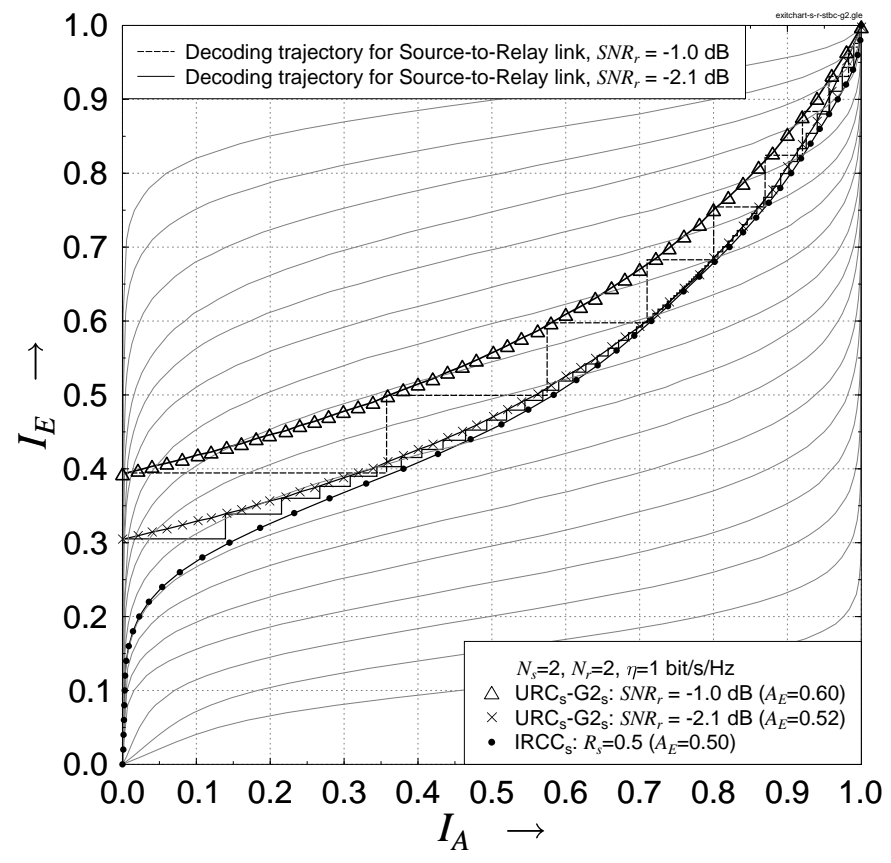

Fig. 5. The EXIT chart curves of the $\mathrm{URC}_{s}-\mathrm{G} 2_{s}$, the $\mathrm{IRCC}_{s}$ with optimized weighting coefficients $\left[\alpha_{1}, \ldots, \alpha_{17}\right]=[0,0,0,0,0,0.327442,0.186505$, $0.113412,0,0.0885527,0,0.0781214,0.0962527,0.0114205,0.0346015$, $0.0136955,0.0500168]$ and 17 IRCC subcodes for the $2 \times 2$ source-to-relay communication link where the $\mathrm{SNR}_{r}$ is the receive SNR at the relay node.

In this contribution, we consider an average code rate of $R_{s}=0.5$ for the outer $\mathrm{IRCC}_{s}$ at the source node and the specific relayaided network geometry with $G_{s r}=8$ and $G_{r d}=2$, which satisfies Eq. (5). The EXIT chart of the serial concatenated $\mathrm{IRCC}_{s^{-}} \mathrm{URC}_{s^{-}}$ $\mathrm{STBC}_{s}$ scheme of the source-to-relay link is depicted in Fig. 5, where the decoding trajectories are computed based on a frame length of 250000 bits. The EXIT curve of the outer $\mathrm{IRCC}_{s}$ having optimized weighting coefficients $\alpha_{i}$ as shown in Fig. 5 was constructed using the curve matching algorithm of [13]. As we can see from Fig. 5, a narrow but marginally open EXIT tunnel emerges for the $2 \times 2$ source-to-relay communication link. A receive SNR of about -2.1 $\mathrm{dB}$ is needed in order to attain a decoding convergence to an infinitesimally low BER. Due to the geometrical-gain of the sourceto-relay communication link, the receive SNR at the relay node can be computed as:

$$
\mathrm{SNR}_{r}=\mathrm{SNR}_{t}+10 \log _{10}\left(G_{s r}\right)[\mathrm{dB}],
$$

where $\mathrm{SNR}_{t}$ is the transmit SNR at the source node. Hence, the minimum $\mathrm{SNR}_{t}$ at the source node required for the sake of obtaining a near error-free performance at the relay node is $-11.1 \mathrm{~dB}$. Furthermore, we assume that both the source and relay nodes transmit their signals using the same transmit energy, hence the same transmit SNR. In order to avoid the potentially high computational complexity at the relay node, a wider-than-necessary EXIT tunnel is created in the EXIT chart of Fig. 5 at the receive SNR of $-1.0 \mathrm{~dB}$ at the relay node, which corresponds to a transmit SNR of $-10 \mathrm{~dB}$ based on Eq. (19) at both the source and relay nodes. As clearly seen in the EXIT chart of Fig. 6 at the destination node, after 5 "inner" iterations between the $\mathrm{IRCC}_{s}$ decoder and the amalgamated " $\mathrm{STBC}_{s}$ - $\mathrm{URC}_{s}$ " decoder, the increase of the area $A_{E}$ under the amalgamated " $\mathrm{STBC}_{S^{-}}$ $\mathrm{URC}_{s}-\mathrm{IRCC}_{s}$ " decoder becomes marginal. Hence, we fix the number of "inner" iterations to $I_{i}^{d}=4$ at the destination node. Following the design procedure above, we obtain the resultant matching EXIT curve for the Ir-DHC-ST coding scheme in Fig. 6, where the $\mathrm{IRCC}_{r}$ 


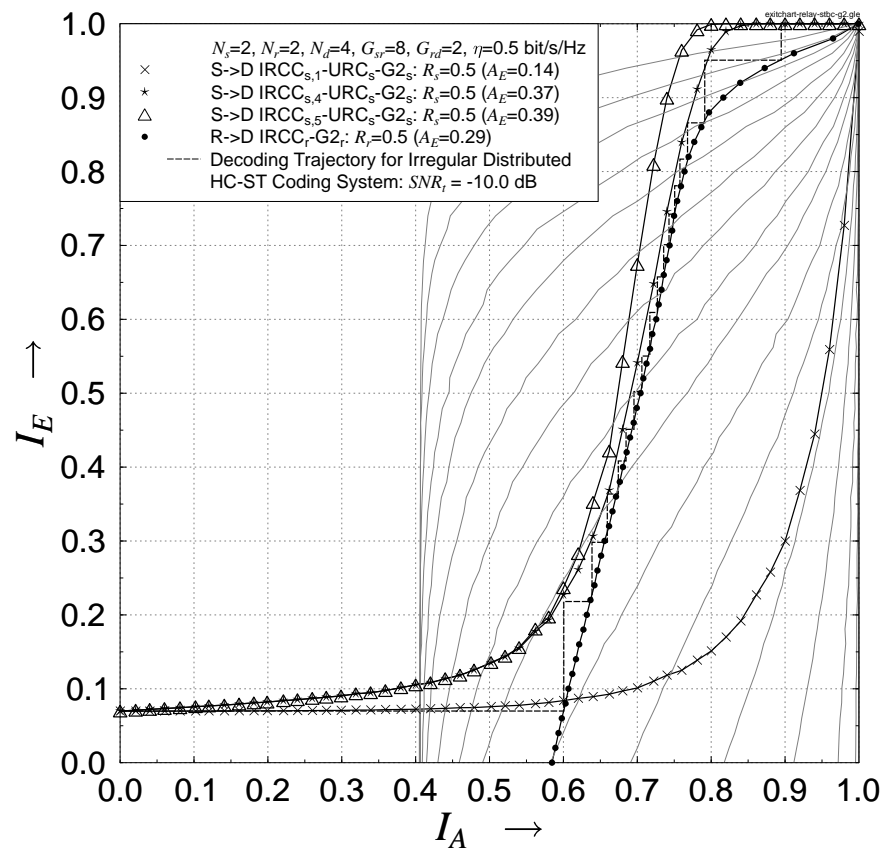

Fig. 6. The EXIT chart curves for the $\mathrm{IRCC}_{s}-\mathrm{URC}_{s}-\mathrm{G} 2_{s}$ with various "inner" iterations, the $\mathrm{IRCC}_{r}-\mathrm{G} 2_{r}$ with $\mathrm{IRCC}_{r}$ having optimized weighting coefficients $\left[\beta_{1}, \ldots, \beta_{17}\right]=[0,0,0,0,0.233115,0.0158742,0.292084$, $0.220065,0.0151108,0,0,0,0,0,0,0,0.22375]$ and 17 SNR-dependent $\mathrm{IRCC}_{r}-\mathrm{G} 2_{r}$ subcodes. The subscript of $\mathrm{IRCC}_{s}$ denotes the number of "inner" iterations between the $\mathrm{IRCC}_{s}$ and " $\mathrm{G} 2_{s}-\mathrm{URC}_{s}$ " decoders and the $\mathrm{SNR}_{t}$ represents the transmit SNR at both the source and relay nodes.

has a maximized average code rate of $R_{r}=0.5$ and the optimized weighting coefficients $\beta_{j}$ are also summarized in Fig. 6. It is clearly seen in Fig. 6 that a narrow-but-open EXIT-tunnel emerges, which indicates the possibility of achieving decoding convergence to an infinitesimally low BER at near-network-capacity SNRs for the IrDHC-ST coding scheme. This prediction is verified in Fig. 6 by plotting the corresponding Monte-Carlo simulation-related decoding trajectory, which indeed reaches the $(1.0,1.0)$ point of the EXIT chart. On the other hand, since the IRCCs at the source and relay nodes have the same code rate of 0.5 , we have $L_{s}=L_{r}$. Hence the effective network throughput is $\frac{L_{s}}{L_{s}+L_{r}} R_{s} \log _{2} 4=0.5 \mathrm{bit} / \mathrm{s} / \mathrm{Hz}$ when 4QAM modulation is employed. According to Eq. (15), we derive the corresponding relay-aided network's DCMC capacity curve for the two-hop cooperative network in Fig. 4, where the network's capacity curve of the unrestricted Continuous-input Continuousoutput Memoryless Channel (CCMC) [9], [10] is also depicted for comparison.

\section{Simulation Results and Discussions}

In this section, we present the BER versus transmit SNR performance of both the perfect and imperfect relaying aided Ir-DHC-ST schemes and non-cooperative IRCC-URC-STBC scheme in Fig. 7. For the Ir-DHC-ST coding scheme, according to the trajectory predictions seen in Figs. 5 and 6, the number of decoding iterations between the $\operatorname{IRCC}_{s}$ decoder and the amalgamated "STBC $\mathrm{S}_{s} \mathrm{URC}_{s}$ " decoder was fixed to $I^{r}=13$ at the relay node. At the destination node, the number of "inner" decoding iterations was fixed to $I_{i}^{d}=4$, while the number of "outer" decoding iterations between the parallel amalgamated " $\mathrm{STBC}_{s}-\mathrm{URC}_{s}$ - $\mathrm{IRCC}_{s}$ " decoder and the amalgamated "STBC $\mathrm{S}_{r}-\mathrm{IRCC}_{r}$ " decoder was fixed to $I_{o}^{d}=17$. On the other hand, for the non-cooperative serial concatenated IRCC-URC-STBC scheme, we employ an outer IRCC, which has the same weighting coefficients $\alpha_{i}$ as that of the $\operatorname{IRCC}_{s}$ in the cooperative system. The number of decoding iterations exchanging extrinsic information between the outer IRCC decoder and the inner "STBC-URC" decoder was fixed to $I_{\text {non }}=13$ as well.

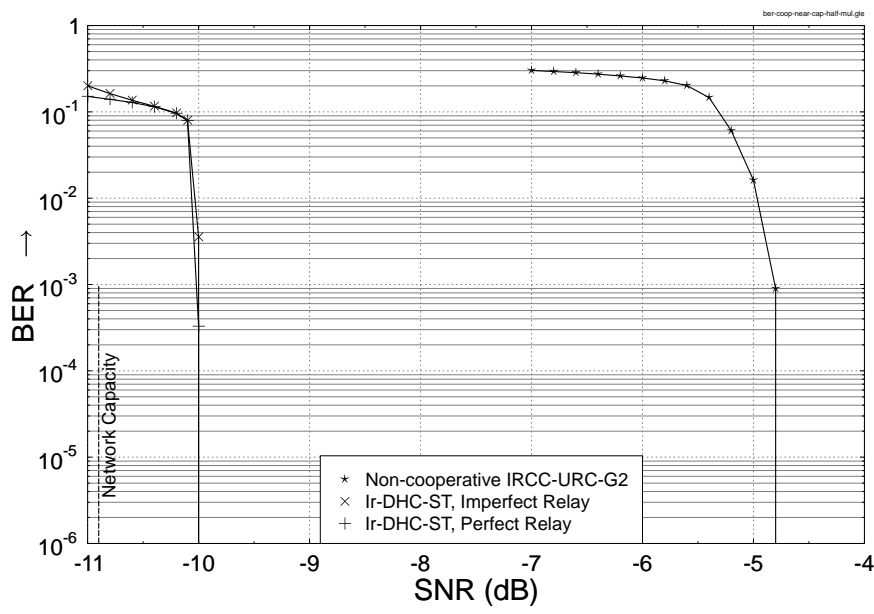

Fig. 7. BER versus transmit SNR performance of both perfect and imperfect relaying Ir-DHC-ST and non-cooperative IRCC-URC-G2 schemes for a frame length of 250000 bits, where the cooperative network is configured with $N_{s}=2, N_{r}=2$ and $N_{d}=4$ and the non-cooperative system is equipped with two transmit antennas and four receive antennas.

As seen in Fig. 7, the Ir-DHC-ST coding scheme outperforms the non-cooperative IRCC-URC-STBC scheme by approximately 5.1 $\mathrm{dB}$ in terms of the required transmit SNR. Since there is a factor $\frac{L_{r}}{L_{s}+L_{r}}=0.5$ multiplexing loss in the half-duplex scenario, this SNR gain corresponds to a $3 \mathrm{~dB}$ lower value of $2.1 \mathrm{~dB}$ in terms of $E_{b} / N_{0}$. On the other hand, the performance of the perfect relaying-aided IrDHC-ST scheme matches the EXIT chart predictions of Fig. 6, while the imperfect relaying-aided Ir-DHC-ST coding scheme performs similarly to the perfect relaying scheme. This is due to the fact that the source information is detected without errors after a sufficiently high number of decoding iterations at the relay node. Furthermore, it is clearly shown in Fig. 7 that the Ir-DHC-ST coding scheme is capable of performing within $(-10.0+3.0)-(-7.9)=0.9 \mathrm{~dB}$ of the corresponding relay-aided network's DCMC capacity in terms of $E_{b} / N_{0}$.

\section{CONCLUSIONS}

In this contribution, we have proposed an Irregular Distributed Hybrid Concatenated Space-Time (Ir-DHC-ST) coding scheme for near-capacity cooperative communications. We derived the DCMC capacity formula of Alamouti's STBC scheme for the two-hop relayaided network. The simulation results show that our joint sourceand-relay mode design based on EXIT chart analysis is capable of near-capacity cooperative communications.

\section{REFERENCES}

[1] A. Sendonaris, E. Erkip, and B. Aazhang, "User cooperation diversity: Part I and II,' IEEE Transactions on Communications, vol. 51, pp. 19271948, Nov. 2003.

[2] J. Laneman, D. Tse, and G. Wornell, "Cooperative diversity in wireless networks: Efficient protocols and outage behavior," IEEE Transactions on Information Theory, vol. 50, pp. 3062-3080, Dec. 2004.

[3] J. Laneman and G. Wornell, "Distributed space-time-coded protocols for exploiting cooperative diversity in wireless networks," IEEE Transactions on Information Theory, vol. 49, pp. 2415-2425, Oct. 2003.

[4] K. Azarian, H. El Gamal, and P. Schniter, "On the achievable diversitymultiplexing tradeoff in half-duplex cooperative channels," IEEE Transactions on Information Theory, vol. 51, pp. 4152-4172, Dec. 2005.

[5] B. Zhao and M. Valenti, "Distributed turbo coded diversity for relay channel," Electronics Letters, vol. 39, pp. 786-787, May 2003.

[6] S. X. Ng, Y. Li and L. Hanzo, "Distributed turbo trellis coded modulation for cooperative communications," in ICC'09, (Dresden, Germany), 14-18 June 2009.

[7] S. ten Brink, "Convergence behaviour of iteratively decoded parallel concatenated codes," IEEE Transactions on Communications, vol. 49, pp. $1727-1737$, Oct. 2001.

[8] S. ten Brink, "Designing iterative decoding schemes with the extrinsic information transfer chart," AEU International Journal of Electronics and Communications, vol. 54, pp. 389-398, Nov. 2000. 
[9] J. G. Proakis, Digital Communications. 4th ed. New York: McGraw-Hill, 2001.

[10] S. X. Ng and L. Hanzo, "On the MIMO channel capacity of multidimensional signal sets," IEEE Transactions on Vehicular Technology, vol. 55, pp. 528-536, March 2006.

[11] S. X. Ng, J. Wang, and L. Hanzo, "Unveiling near-capacity code design: The realization of shannon's communication theory for mimo channels," Communications, 2008. ICC '08. IEEE International Conference on, pp. 1415-1419, May 2008.

[12] L. Kong, S. X. Ng and L. Hanzo, "Near-capacity three-stage downlink iteratively decoded generalized layered space-time coding with low complexity," in GLOBECOM'08, (New Orleans, LA, USA), pp. 1-6, 30 Nov.-04 Dec. 2008.

[13] M. Tüchler and J. Hagenauer, "EXIT charts of irregular codes," in Proceeding of the 36th Annual Conference on Information Sciences and Systems [CDROM], (Princeton, NJ, USA), March 2002.

[14] M. Tüchler, "Design of serially concatenated systems depending on the block length," IEEE Transactions on Communications, vol. 52, pp. 209218, Feb. 2004

[15] H. Ochiai, P. Mitran, and V. Tarokh, "Design and analysis of collaborative diversity protocols for wireless sensor networks," IEEE 60th Vehicular Technology Conference, 2004 Fall, vol. 7, pp. 4645-4649 Vol. 7, Sept. 2004

[16] A. Host-Madsen and J. Zhang, "Capacity bounds and power allocation for wireless relay channels," IEEE Transactions on Information Theory, vol. 51, pp. 2020-2040, June 2005.

[17] A. Host-Madsen, "Capacity bounds for cooperative diversity," IEEE Transactions on Information Theory, vol. 52, pp. 1522-1544, April 2006.

[18] S. M. Alamouti, "A simple transmit diversity technique for wireless communications," IEEE Journal on Selected Areas in Communications, vol. 16, pp. 1451-1458, Oct. 1998.

[19] D. Divsalar, S. Dolinar and F. Pollara, "Serial turbo trellis coded modulation with rate-1 inner code," in ISIT, (Sorrento, Italy), p. 194, 25-30 June 2000.

[20] L. Hanzo, T. H. Liew and B. L. Yeap, Turbo Coding, Turbo Equalisation and Space Time Coding for Transmission over Wireless channels. New York, USA: John Wiley IEEE Press, 2002. 